\title{
Lytic Bone Metastasis in a Case with Primary Breast Lymphoma
}

\author{
Yasemin Benderli Cihan ${ }^{1, *}$, Alaettin Arslan ${ }^{1}$, Mehmet Faik Cetindag ${ }^{2}$ \\ ${ }^{1}$ Kayseri Education and Research Hospital, Department of Radiation Oncology, Turkey \\ ${ }^{2}$ Ankara Education and Research Hospital, Department of Radiation Oncology, Turkey
}

Copyright $(\mathcal{C} 2015$ by authors, all rights reserved. Authors agree that this article remains permanently open access under the terms of the Creative Commons Attribution License 4.0 International License

\begin{abstract}
Bone metastasis alone without involvement of bone marrow is rarely seen in primary breast lymphoma (PBL). It has a poor prognosis. A 64-years old woman was diagnosed as high-grade PBL T type. The patient received 6 sessions of chemotherapy with a diagnosis of stage IIAE PBL. The patient achieved remission and was followed for 12 years. She underwent surgery due to fracture at lower half of right femur. Palliative radiotherapy (30 Gy) was given to right femur. Here, we presented a patient with high-grade PBL $\mathrm{T}$ type developed lytic bone metastasis without bone marrow involvement.
\end{abstract}

Keywords Primary Breast Lymphoma, Radiotherapy, Lytic Bone Metastases

\section{Introduction}

Primary breast lymphoma is defined as lymphoma at breast or axillary region alone without involvement at any other region of the body. It comprises $0.04-0.5 \%$ of all breast cancers and $1.7-2.2 \%$ of extra-nodal lymphomas [1]. It has an increasing incidence by time. It is usually seen at sixth decade and is more frequent in female gender. The most common histological type is diffuse, large B-cell lymphoma. High-grade $\mathrm{T}$ type is seen in rare cases. Bone marrow involvement is present in $13 \%$ of all cases of primary breast lymphoma. Generally, Ann Arbor staging system is used for staging [2-4].

There is no established standard therapy for PBL. As in the lymphomas at other regions, it is treated by using anthracycline-based systemic chemotherapy and radiotherapy $[1,5,6]$. Five-year survival rate varies between $45 \%$ and $70 \%$. Its prognosis is similar to those seen at other regions and depends on cellular type, clinical stage and treatment $[1,3,5]$.

In this manuscript, we presented clinical characteristics, therapeutic approaches and outcomes in a patient who had been treated with high-grade, PBL $\mathrm{T}$ type with lytic bone metastasis without bone marrow involvement.

\section{Case Report}

A 64-years old woman presented with a palpable mass in June, 2001. Left modified radical mastectomy was performed, as fine-needle biopsy was reported as malign pathology. On immunohistochemical evaluation, there was diffuse LCA and CD 45RO staining and positive CD 79 staining in large areas in the surgical specimen. In surgical specimen, there was an involvement in one axillary lymph node. Thus, the case was considered as high-grade $\mathrm{T}$ cell lymphoma based on histopathological appearance and staining characteristics (Figure 1). No regional or distant organ involvement was detected on physical examination and radiological evaluations (computerized tomography scans of thorax, neck, brain and abdominal). Bone marrow biopsy was interpreted as normal. Since there was no disease finding in other regions, the patient was considered as primary breast lymphoma. The patient received 6 session of CHOP regime (cyclophosphamide, doxorubicin, vincristine, prednisone) with a diagnosis of stage IIAE PBL. Control examinations were normal after primary treatment. No recurrence or metastasis was detected.

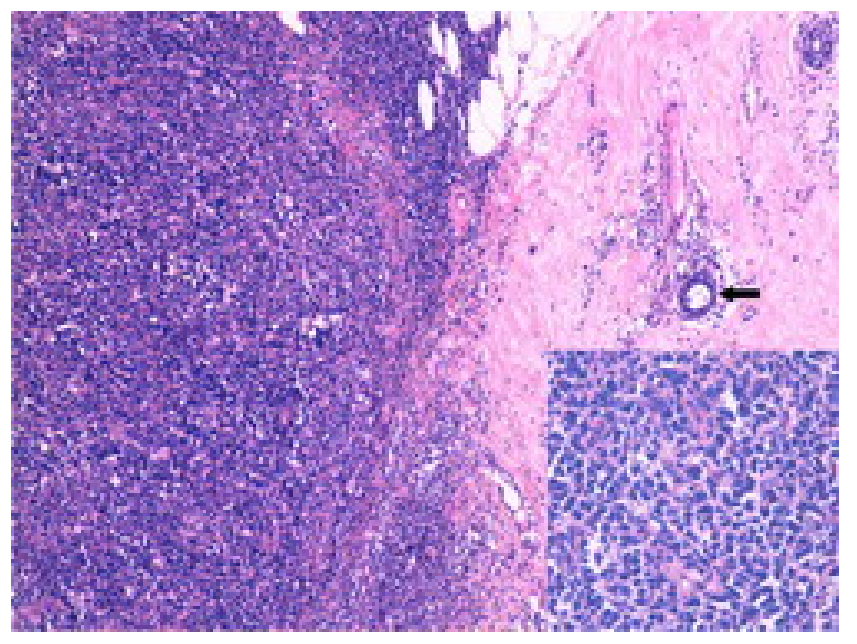

Figure 1. Hematoxylin and eosin-stained section shows infiltrate of atypical lymphocytes with normal breast tissue being identified (100x). 
A fracture at distal half of right femur developed 12 years after diagnosis (Figure 2). The patient was consulted to our department after surgery due to fracture. Perioperative bone marrow aspiration was considered as normocellular. On physical examination, no lymphadenopathy was detected at cervical and axillary region. Respiratory and cardiovascular examinations were considered as normal. On CT scans of neck, thorax or abdomen, no lymphadenopathy or mass was detected. On whole-body bone scintigraphy, increased uptake was detected at distal half of right femur. Palliative radiotherapy (overall $30 \mathrm{~Gy}$ in 10 fractions) was given to distal half of right femur 4 weeks after surgery. CBC was found as normal after radiotherapy. Biochemical parameters other than LDH (478 IU; normal range: 140-280 IU) was normal. On PET-CT scan, intense FDG uptake was detected in multiple lymph nodes at right distal paratracheal area, aortopulmonary window and bilateral hilar area (largest being $14 \times 9 \mathrm{~mm}$ in size), right obturator and inguinal areas (largest being $21 \times 12 \mathrm{~mm}$ in size) were detected, while hyper-intense FDG uptake at distal half of right femur and proximal metaphysodiaphyseal area area of left tibia in musculoskeletal scanning. No involvement was found at central nervous system (CNS) (Figure 3). Chemotherapy was scheduled as organ involvement was predominant.

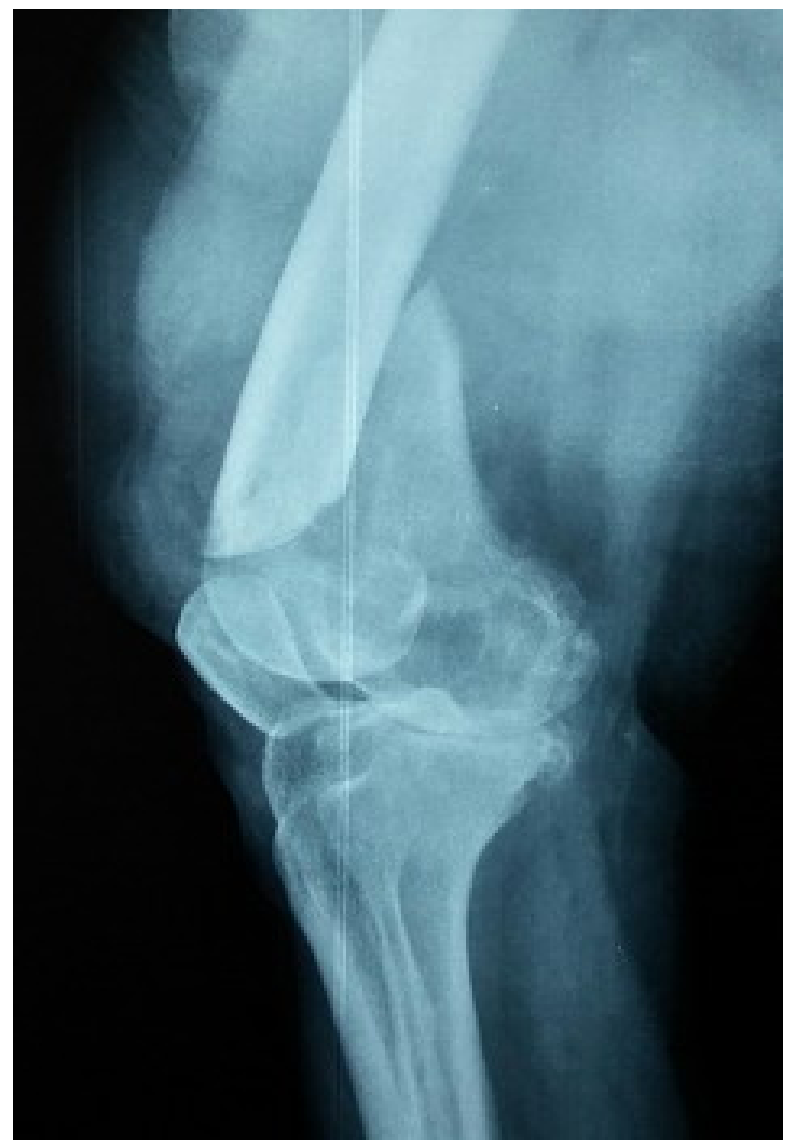

Figure 2. Right femur pathologic fracture

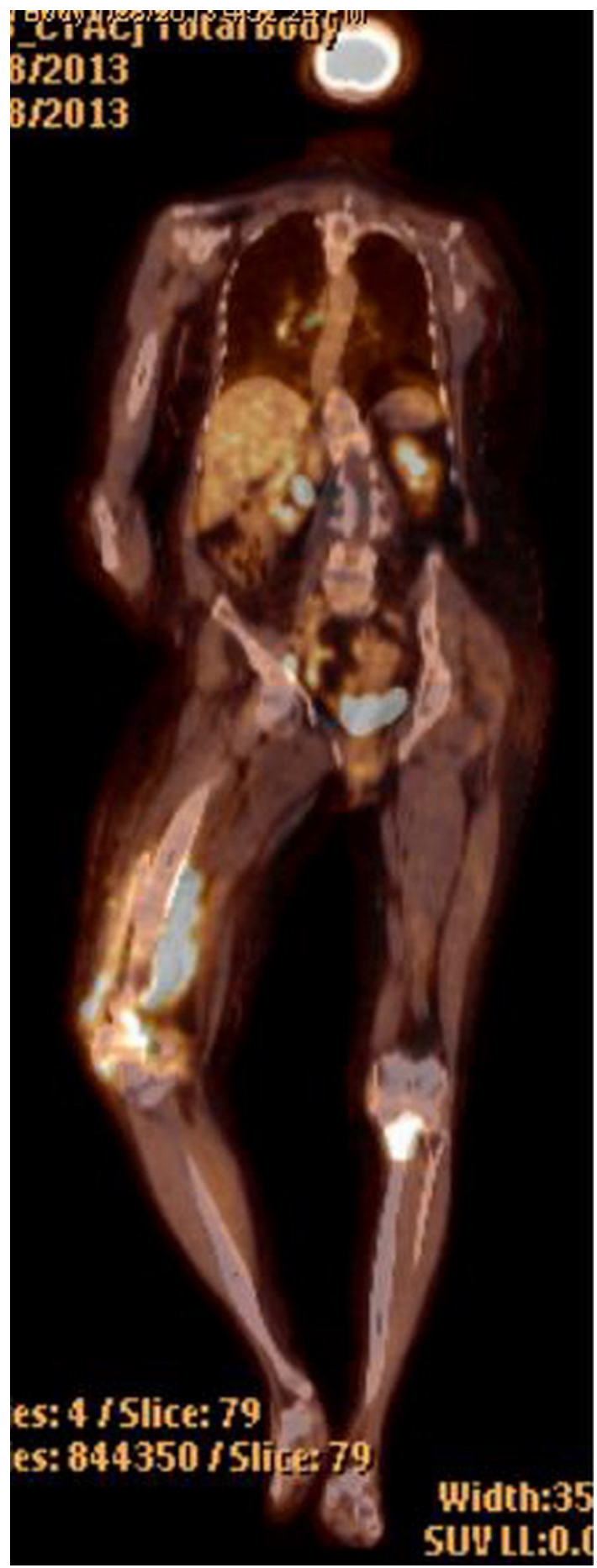

Figure 3. PET-CT scan intense FDG uptake

\section{Discussion}

The behavior of primary breast lymphoma is similar to the lymphomas rising from other regions with identical age and histology. Majority of patients present with unilateral, painless, palpable mass. It is usually encountered at upper outer quadrant of right breast and it is more frequent in female gender. B symptoms are lacking in most cases (82-96\%) [1,6,7]. Diagnostic approach should involve 
complete blood count, biochemical parameters, LDH measurement, bone marrow aspiration/biopsy, mammography, CT scans of thorax, abdomen and pelvis, and PET-CT, if indicated. Role of mammography is limited in radiological diagnosis. Sonographic evaluation has more specific characteristics $[7,8,9]$. Our case presented with palpable mass localized as upper outer quadrant of right breast. She had no B symptoms.

Diffuse, large B cell lymphoma is most commonly observed histological type. In addition, follicular lymphoma, MALT lymphoma and Burkitt lymphoma can also be observed. T cell type is seen in rare cases [3-9]. In a study by Kuper-Hommel et al., it was reported that incidence of diffuse, large B cell lymphoma varied between $45 \%$ and $79 \%$ [10]. Authors also reported that this type is generally unilateral and often observed in middle-age women [10]. In a meta-analysis including 450 cases, Jennings et al. reported that the most common type was diffuse, large cell lymphoma with a frequency of 53\% [4]. In our case, tumor histology was compatible with high-grade $\mathrm{T}$ cell lymphoma and immunohistochemical staining supported $\mathrm{T}$ cell subtype.

Management of primary breast lymphoma is similar to those in other lymphoma types, including surgery, chemotherapy, and radiotherapy [3-6]. As primary breast lymphoma usually tends to persist confined, mastectomy isn't recommended in these patients and breast-sparing surgery is preferred in such cases other than those with bulky disease and aggressive histopathology [4,10-13]. In a systematic review on 465 patients, Jenning et al. reported that mastectomy didn't improve survival and local control in PBL [4].

When radiotherapy and chemotherapy alone were compared to chemoradiotherapy, there was a significant difference in disease-free and overall survival rates in favor of combined therapy. In a study on 96 patients with early stage PBL (stage I and II) by Aviles et al., radiotherapy (45 Gy) was given to 30 patients while chemotherapy to 32 patients and combined treatment to 30 patients (30 Gy). Complete response was achieved in 20 patients in radiotherapy arm; in 19 patients in chemotherapy arm; and in 30 patients in combined treatment arm. Ten-year disease-free survival rates were $50 \%, 57 \%$ and $83 \%$ whereas overall survival rates were $50 \%, 50 \%$ and $76 \%$, respectively [4]. A study on 204 patients by Ryan et al., a multicenter study on 36 patients by Martinelli et al . reported similar outcomes $[3,7,8]$. In a single-center study on 45 patients by Validire et al., histological type was diffuse, large B cell lymphoma in 38 patients $(83 \%)$. Other histological types included lymphocytic lymphoma in 2 patients, grade I follicular NHL in 3 patients, MALT lymphoma in one patient and grade IIIB follicular lymphoma in another patient. In that study, authors analyzed 38 patients with diffuse, large B cell lymphoma and classified 37 patients with available data according to International Prognostic Index as follows: 19 patients $(51 \%)$ in low-risk group, 5 patients $(14 \%)$ in low-moderate risk group, 6 patients (16\%) in moderate-high risk group, and 7 patients (19\%) in high risk group. Authors observed that there was worsening in 18 patients (47\%) and worsening in CNS in 3 patients during median follow-up of 96-months. Five-year disease-free and overall survival rates were calculated as $54 \%$ and $61 \%$, respectively [5]. As a result, studies concluded that chemotherapy alone or chemotherapy plus radiotherapy should be given to patients with moderate and high grade disease. It was suggested that radiotherapy improved local control with recommended dose of 40 Gy (35-50 Gy). Today, CHOP is most commonly used chemotherapy regime in such patients $[4,9,12,13]$. It is unclear whether rituximab should be added [9]. In our case, modified radical mastectomy was performed when fine-needle aspiration biopsy was reported as malign disease. Excisional biopsy could prevent unnecessary mastectomy, if performed in our case. The patient received 6 sessions CHOP due to detection of axillary involvement in pathological evaluation. Disease-free survival was 12 years in our patient although no radiotherapy was given.

In the literature, Validire et al. reported bone marrow involvement of PBL [5]. To best of our knowledge, there is no information regarding bone metastasis. PBL is considered within NHL group. Lymphamatous involvement of musculoskeletal system is rare in NHLs. Lymphomas are not typically associated with lytic bone lesions. Lytic bone metastasis is generally seen metastatic breast cancer and multiple myeloma. In general, bone metastasis in lymhoma is observed following bone marrow involvement and seen at femur and pelvis. The primary complaint is pain in such patients. Patients suffer from acute, rapidly worsening pain which doesn't relieve with rest. Pathological fractures in this area results in impaired mobility, dysfunction and decreased quality of life [14].

Our case presented with pathological fracture after prolonged disease-free period. Bone marrow aspiration biopsy was normal. There was intense FDG uptake on PET-CT and increased pathological activity uptake in whole-body bone scintigraphy.

\section{Conclusions}

High-grade, primary breast lymphoma $\mathrm{T}$ type with lytic bone metastasis without bone marrow involvement is rarely encountered in lymphoma. Chemotherapy or radiotherapy is recommended in the treatment

\section{REFERENCES}

[1] Jeanneret-Sozzi W, Taghian A, Epelbaum R, et al. Primary breast lymphoma: patient profile, outcome and prognostic factors. A multicentre Rare Cancer Network study. BMC Cancer 2008 1; 8: 86.

[2] Avilés A, Delgado S, Nambo MJ,et al. Primary breast 
lymphoma: results of a controlled clinical trial. Oncology 2005; 69(3): 256-60.

[3] Ryan G, Martinelli G, Kuper-Hommel M,et al. Primary diffuse large B-cell lymphoma of the breast: prognostic factors and outcomes of a study by the International Extranodal Lymphoma Study Group. Ann Oncol 2008; 19(2): 233-41.

[4] Jennings WC, Baker RS, Murray SS, et al. Primary breast lymphoma: the role of mastectomy and the importance of lymph node status. Ann Surg 2007; 245(5): 784-9.

[5] Validire P, Capovilla M, Asselain B et al. Primary breast non-Hodgkin's lymphoma: a large single center study of initial characteristics, natural history, and prognostic factors. American Journal of Hematology, 2009; 84(3): 133-139.

[6] Yhim HY, Kang HJ, Choi YH, et al. Clinical outcomes and prognostic factors in patients with breast diffuse large B cell lymphoma; Consortium for Improving Survival of Lymphoma (CISL) study. BMC Cancer 2010; 10: 321.

[7] Ganjoo K, Advani R, Mariappan MR, et al. Non-Hodgkin lymphoma of the breast. Cancer 2007; 110(1): 25-30.

[8] Martinelli G, Ryan G, Seymour JF et al. Primary follicular and marginal-zone lymphoma of the breast: clinical features, prognostic factors and outcome: a study by the International
Extranodal Lymphoma Study Group. Annals of Oncology, 2009; 20(12): 1993-1999.

[9] Aviles A, Castaneda C, Neri N et al. Rituximab and dose dense chemotherapy in primary breast lymphoma. Haematologica 2007; 92(08): 1147-8.

[10] Kuper-Hommel MJJ, Snijder S, Janssen-Heijnen MLG et al. Treatment and survival of 38 female breast lymphomas: a population-based study with clinical and pathological reviews. Ann Hematol 2003; 82: 397-404

[11] Lin Y, Guo XM, Shen KW et al. Primary breast lymphoma: Long-term treatment outcome and prognosis. Leukemia \& Lymphoma 2006; 47(10): 2102-9

[12] Wong WW, Schild SE, Halyard MY et al. Primary Non-Hodgkin Lymphoma of the Breast: The Mayo Clinic Experience. Journal of Surgical Oncology 2002; 80: 19-25.

[13] Radkani Pejman, Joshi D, Paramo JC, et al. Primary breast lymphoma: 30 years of experience with diagnosis and treatment at a single medical center. JAMA Surg. Published online November 20, 2013. doi:10.1001/jamasurg.2013.2283

[14] Ali H, Yeku O, Giesler D, et al. Breast mass and lytic bone lesions: A rare presentation of Non-Hodgkin's Lymphoma arising in the breast. Case Rep Oncol Med. 2013;2013:547171. doi: 10.1155/2013/547171. Epub 2013 Oct 21 . 